\title{
UPPER BOUND OF MULTIPLICITY OF F-PURE RINGS
}

\author{
CRAIG HUNEKE AND KEI-ICHI WATANABE \\ (Communicated by Irena Peeva)
}

ABstract. This paper answers in the affirmative a question raised by Karl Schwede concerning an upper bound on the multiplicity of F-pure rings.

\section{INTRODUCTION}

In the problem session of the workshop at AIM, August 2011, titled "Relating Test Ideals and Multiplier Ideals", Karl Schwede, inspired by the work of Stefan Helmke $\mathrm{He}$, posed the following question.

Let $(R, \mathfrak{m})$ be a Noetherian local ring of characteristic $p>0$ of dimension $d$ and embedding dimension $v$. Assume that $R$ is F-pure. Then does the multiplicity $e(R)$ of $R$ always satisfy

$$
e(R) \leq\left(\begin{array}{c}
v \\
d
\end{array}\right) ?
$$

We will prove this inequality is true and follows from a Briançon-Skoda type theorem. Our results can be used to give an alternate proof of one of the main results of $[\mathrm{ST}]$, bounding the number of F-pure centers in an F-pure F-finite local ring, which was one of the reasons for asking the motivating question. See Remark 3.4 .

\section{Preliminaries}

Let $(R, \mathfrak{m})$ be either a Noetherian local ring or $R=\bigoplus_{n \geq 0} R_{n}$ be a graded ring finitely generated over a field $R_{0}=k$. We always assume that either $R$ contains a field of characteristic $p>0$ or $R$ is essentially of finite type over a field of characteristic 0 . We always assume that our ring $R$ is reduced.

Definition 2.1. Let $R$ have characteristic $p$. We denote by $R^{\circ}$ the set of elements of $R$ that are not contained in any minimal prime ideal. The tight closure $I^{*}$ of $I$ is defined to be the ideal of $R$ consisting of all elements $x \in R$ for which there exists $c \in R^{\circ}$ such that $c x^{q} \in I^{[q]}$ for all large $q=p^{e}$.

Definition 2.2. We say that a local ring $(R, \mathfrak{m})$ is $F$-rational if it is a homomorphic image of a Cohen-Macaulay ring and for every parameter ideal $J$ of $R$ we have $J^{*}=J$. It is known that F-rational rings are normal and Cohen-Macaulay.

Received by the editors June 27, 2013 and, in revised form, August 29, 2013.

2010 Mathematics Subject Classification. Primary 13H15; Secondary 13C99, 14 B05.

The first author was partially supported by NSF grant 1259142 .

The second author was partially supported by Grant-in-Aid for Scientific Research 20540050 and Individual Research Expense of College of Humanity and Sciences, Nihon University.

The authors thank AIM for the opportunity to work together. 
Definition 2.3. Assume that $R$ contains a field of characteristic $p>0$ and $q=p^{e}$ is a power of $p$.

(1) For a power $q=p^{e}$ and ideal $I$ in $R$, we denote by $I^{[q]}$ the ideal generated by $\left\{a^{q} \mid a \in I\right\}$.

(2) We write $R^{1 / q}$; then we say that $R$ is $F$-pure if for every $R$-module $M$, the natural map $M=M \otimes_{R} R \rightarrow M \otimes_{R} R^{1 / p}$, sending $x \in M$ to $x \otimes 1$, is injective.

(3) Let $I$ be an ideal of $R$ and $x \in R$. If $R$ is F-pure and if $x^{q} \in I^{[q]}$, then $x \in I$. This follows from (2) if we put $M=R / I$.

\section{The MAin RESUlts}

The following theorem is our main result in this article.

Theorem 3.1. Let $(R, \mathfrak{m})$ be a Noetherian local ring with $\operatorname{dim} R=d$ and embedding dimension $v$. Then:

(1) If $R$ is a rational singularity or F-rational, then $e(R) \leq\left(\begin{array}{c}v-1 \\ d-1\end{array}\right)$.

(2) If $R$ is F-pure, then $e(R) \leq\left(\begin{array}{c}v \\ d\end{array}\right)$.

This theorem easily follows from the following theorem. We recall that a reduction $J$ of an ideal $I$ is an ideal $J \subset I$ such that for large $n, I^{n}=J I^{n-1}$. A minimal reduction is a reduction minimal with respect to inclusion. A fundamental fact is that in a Noetherian local ring of dimension $d$ having infinite residue class field, minimal reductions are always generated by at most $d$ elements. See [HS, Section 8.3].

Theorem 3.2. Let $(R, \mathfrak{m})$ be a Noetherian local ring with $\operatorname{dim} R=d$ and let $J \subset \mathfrak{m}$ be a minimal reduction of $\mathfrak{m}$.

(1) If $R$ is a rational singularity or F-rational, then $\mathfrak{m}^{d} \subset J$.

(2) If $R$ is F-pure, then $\mathfrak{m}^{d+1} \subset J$.

Proof. Statement (1) is well known and follows from a Briançon-Skoda type theorem (cf. [HH], LT] $)$.

For statement (2) we will prove the following statement.

Assume $R$ is F-pure and $I$ is an ideal generated by $r$ elements, which contains a non-zerodivisor; then $\overline{I^{r+1}} \subset I$. This is sufficient to prove Theorem 3.2 since $\mathfrak{m}^{d+1} \subset \overline{\mathfrak{m}^{d+1}}=\overline{J^{d+1}}$.

Now, take $x \in \overline{I^{r+1}}$. Then we can take $c \in R^{\circ}$ such that for sufficiently large $N, c x^{N} \in I^{(r+1) N}$. Then $c x^{N} \in c\left(I^{(r+1) N}: c\right)$. The latter is contained in $c R \cap$ $I^{(r+1) N}$ and by the Artin-Rees Lemma, there exists $k$ such that $c R \cap I^{(r+1) N} \subset$ $c I^{(r+1) N-k}$ for sufficiently large $N$. Now, we have shown that $c x^{N} \in c I^{(r+1) N-k}$. Note that $I^{r q} \subset I^{[q]}$. Taking sufficiently large $N=q=p^{e}$ and noting that $c$ is a non-zerodivisor, we get $x^{q} \in I^{[q]}$. Since $R$ is F-pure, we get $x \in I$. This was proved in [Hu], Proposition 4.9, when $R$ is Cohen-Macaulay.

It is easy to prove Theorem 3.1 using Theorem 3.2 .

Proof of Theorem $3.2 \Longrightarrow$ Theorem 3.1 . We have the following inequality and the equality holds if and only if $R$ is Cohen-Macauly (cf. $\mathrm{BH}$, Corollary 4.7,11):

$$
e(R) \leq l_{R}(R / J) \text {. }
$$


So, it suffices to show that $l_{R}(R / J)$ is bounded by the right-hand side of the inequalities in Theorem 3.1. Now, let $x_{1}, \ldots, x_{d}, y_{1}, \ldots, y_{v-d}$ be minimal generators of $\mathfrak{m}$ with $J=\left(x_{1}, \ldots, x_{d}\right)$. Then $R / J$ is generated by the monomials of $y_{1}, \ldots, y_{v-d}$ of degree $\leq d-1$ (resp. degree $\leq d$ ) in case (1) (resp. case (2)) by Theorem 3.2. It is easy to see that the number of monomials of $y_{1}, \ldots, y_{v-d}$ of degree $\leq d-1$ (resp. degree $\leq d)$ is $\left(\begin{array}{c}v-1 \\ d-1\end{array}\right)\left(\operatorname{resp} .\left(\begin{array}{l}v \\ d\end{array}\right)\right)$.

Remark 3.3. Assume we have equality in Theorem 3.1(1) or (2). Then $R$ is CohenMacaulay since we must have equality in (3.1.1), too. Moreover, since the associated graded ring of $R$ has the same embedding dimension and multiplicity with $R$, $\operatorname{gr}_{\mathfrak{m}}(R)$ is also Cohen-Macaulay in this case.

Remark 3.4. Another class of singularities which would be natural to consider are F-injective (respectively, Du Bois) singularities. So, it is natural to ask if Theorem 3.2 is true if we assume $R$ is F-injective or a Du Bois singularity. We do not know the answer. Also, we point out that our Theorem 3.2 (2) gives another proof of one of the main theorems of [ST] concerning the number of F-pure centers. Actually, in Theorem 5.10 of $[\mathrm{ST}]$, if $Q_{i}(1 \leq i \leq N)$ are prime ideals of dimension $d$ of $R$ such that every $R / Q_{i}$ is F-pure, then certainly the number $N$ is bounded by the multiplicity of $R /\left[\bigcap_{i=1}^{N} Q_{i}\right]$ and the latter is F-pure. Thus we can apply our Theorem $3.2(2)$. We thank the anonymous referee for suggesting these points.

\section{Actual upper bound}

The upper bound in Theorem 3.1 (2) is taken by the following example.

Example 4.1. Let $\Delta$ be a simplicial complex on the vertex set $\{1,2, \ldots, v\}$, whose maximal faces are all possible $d-1$ simplices. Then the Stanley-Reisner $\operatorname{ring} R=$ $k[\Delta]$ has dimension $d$ and $e(R)=\left(\begin{array}{c}v \\ d\end{array}\right)$. Note that Stanley-Reisner rings are always F-pure.

Remark 4.2. (1) Are there other examples where we have equality in Theorem 3.1 (2) if $v \geq d+2$ ? It is shown in GW that in the case of $d=1$, this is the only example if we assume $(R, \mathfrak{m})$ is a complete local ring with algebraically closed residue field.

(2) It is natural to ask if there are examples where we have equality in Theorem 3.1 (1) if $v \geq d+2$ and $d \geq 3$. If $d=2$, we have always $e(R)=v-1$ (cf. [Li]). See remark (4) below.

(3) It is not difficult to see that the examples which attain the maximal value in Theorem 3.1 (1) must be generated by $\left(\begin{array}{c}v-1 \\ d\end{array}\right)$ elements of degree $d$, and have defining ideal with a linear resolution. In fact, let $R$ be a rational singularity or F-rational having maximal multiplicity $e(R)=\left(\begin{array}{c}v-1 \\ d-1\end{array}\right)$, where the dimension of $R$ is $d$ and $v$ is the embedding dimension. Let $A$ be a general Artinian reduction of $R$, that is to say, $A$ is $R$ modulo a general linear system of parameters (we assume infinite field here). Then as the proof of the main theorem shows, $A \cong$ $k\left[x_{1}, \ldots, x_{c}\right] /\left(x_{1}, \ldots, x_{c}\right)^{d}$, where $c=v-d$ is the embedding codimension of $R$. It is well known that $\left(x_{1}, \ldots, x_{c}\right)^{d}$ has a linear resolution. Since $R$ must be CohenMacaulay, its defining ideal will also have a linear resolution. Observe that the 
$a$-invariant of $R$ must then be -1 , since the dimension of $R$ is $d$, and the socle degrees of the Artinian reduction $A$ are all $d-1$.

The converse will often be true; if $S$ is a polynomial ring, and $R^{\prime}$ a graded Frational quotient ring (or rational singularity) whose defining ideal $J$ has a linear resolution, then provided the common degree of the generators of $J$, say $d$, is at most the dimension of $R^{\prime}$, then one should be able to cut $R^{\prime}$ down by general linear forms (at least over an algebraically closed field) to a ring $R$ such that the dimension of $R$ is exactly $d$ and $R$ is F-rational or has rational singularities on the punctured spectrum (see, for example, [SZ]). But then it is enough to check that the $a$-invariant is negative to prove $R$ is F-rational $\mathrm{Wa}, \mathrm{F}$. This follows from the fact $R$ has a linear resolution over the polynomial ring obtained from $S$ by cutting with the same general linear forms. The $a$-invariant of $R$ will be -1 . The multiplicity of $R$ will be exactly $\left(\begin{array}{c}v-1 \\ d-1\end{array}\right)$, where $v$ is the embedding dimension of $R$.

(4) To see an explicit example as in (3), with even an isolated singularity, consider the ideal of maximal minors of a generic $r$ by $s$ matrix $X$ over an algebraically closed field of characteristic 0 . Assume that $2 r \leq s+3$. Let $S$ be the ambient polynomial ring, and let $R^{\prime}=S / I$, where $I$ is generated by the maximal minors of the generic matrix $X$. The singular locus of $R^{\prime}$ is defined by the image of the $r-1$ size minors of $X$, which has height in $R^{\prime}$ exactly $(r-(r-1)+1)(s-(r-1)+1)-(s-r+1)=$ $2(s-r+2)-(s-r+1)=s-r+3$. Since $r \leq s-r+3$ by assumption, we can reduce $R^{\prime}$ modulo $r s-s+2 r-1$ general linear forms to reach an $r$-dimensional ring $R$ with an isolated singularity (see [F2, Satz 5.2]), defined by the maximal minors of an $r$ by $s$ matrix $Y$ of linear forms such that the $r$ by $r$ minors have generic height. The $a$-invariant of $R$ is -1 , so by the results of [F] and [Wa], $R$ has a rational singularity. The multiplicity of $R$ is exactly $\left(\begin{array}{c}v-1 \\ d-1\end{array}\right)$, where $v$ is the embedding dimension and $d$ is the dimension of $R$.

It seems likely that the following question will have a positive answer:

Question 4.3. Assume that $(R, \mathfrak{m})$ is a rational singularity or F-rational with dimension $d$ and embedding dimension $v$ with maximal possible multiplicity (as in Theorem 3.1). Then is the associated graded ring $g r_{\mathfrak{m}}(R)$ Cohen-Macaulay having a defining ideal with linear resolution?

\section{Case of Gorenstein Rings}

If $R$ is Gorenstein, the upper bound is largely reduced by the duality. We prove:

Theorem 5.1. Let $(R, \mathfrak{m})$ be a Gorenstein Noetherian local ring with $\operatorname{dim} R=d$ and embedding dimension $v$.

(1) If $R$ is a rational singularity or F-rational with $\operatorname{dim} R=2 r+1$, then $e(R) \leq$ $\left(\begin{array}{c}v-r-1 \\ r\end{array}\right)+\left(\begin{array}{c}v-r-2 \\ r-1\end{array}\right)$.

(2) If $R$ is a rational singularity or F-rational with $\operatorname{dim} R=2 r$, then $e(R) \leq$ $2\left(\begin{array}{c}v-r-1 \\ r-1\end{array}\right)$.

(3) If $R$ is F-pure with $\operatorname{dim} R=2 r+1$, then $e(R) \leq 2\left(\begin{array}{c}v-r-1 \\ r\end{array}\right)$. 
(4) If $R$ is F-pure with $\operatorname{dim} R=2 r$, then $e(R) \leq\left(\begin{array}{c}v-r \\ r\end{array}\right)+\left(\begin{array}{c}v-r-1 \\ r-1\end{array}\right)$.

Proof. We will prove the first statement. All the others follow in exactly the same manner. We may assume that the residue field is infinite. Let $J$ be a minimal reduction of the maximal ideal of $R$, and let $B=R / J$, an Artinian Gorenstein ring. By Theorem [3.2. we know that $\mathfrak{m}^{d} \subset J$, so that if $\mathfrak{n}$ denotes the maximal ideal of $B, \mathfrak{n}^{2 r+1}=0$. We estimate the length of the $B$ (which is the multiplicity of $R)$. First, observe that since $B$ is Gorenstein, $l\left(B / \mathfrak{n}^{t}\right)$ is the same as the length of the Matlis dual module, $\operatorname{Hom}\left(B / \mathfrak{n}^{t}, B\right)$, which is equal to the length of $\left(0: \mathfrak{n}^{t}\right)$. Therefore,

$$
e(R)=l(B)=l\left(\mathfrak{n}^{r}\right)+l\left(B / \mathfrak{n}^{r}\right) \leq l\left(\left(0: \mathfrak{n}^{r+1}\right)\right)+l\left(B / \mathfrak{n}^{r}\right)=l\left(B / \mathfrak{n}^{r+1}\right)+l\left(B / \mathfrak{n}^{r}\right),
$$

where the inequality follows because $\mathfrak{n}^{r} \subset\left(0: \mathfrak{n}^{r+1}\right)$. Now $B$ has embedding dimension $v-(2 r+1)$. As before, $B$ is a homomorphic image of a polynomial ring in $v-(2 r+1)$ variables. As above, the length of a polynomial ring in this many variables modulo the $(r+1)$ st power of its maximal ideal is $\left(\begin{array}{c}v-r-1 \\ r\end{array}\right)$, while modulo the $r$ th power is $\left(\begin{array}{c}v-r-2 \\ r-1\end{array}\right)$, giving the statement of part (1).

Remark 5.2. Again, the upper bound in (3), (4) is taken by the Stanley-Reisner ring of "Cyclic Polytopes" (cf. [St]).

Question 5.3. As was the case in the last section, it is reasonable to ask the following: suppose that $(R, \mathfrak{m})$ is Gorenstein with rational singularity (or F-rational) having the maximal possible multiplicity given the dimension and embedding dimension. Then is $g r_{\mathfrak{m}}(R)$ Gorenstein with "symmetric linear" resolution (i.e., pure resolution with degree sequence $(n, n+1, \ldots, n+s, 2 n+s))$ ?

\section{ACKNOWLEDGEMENT}

The authors are grateful to Naoki Terai for suggesting cyclic polytopes as examples, and to Karl Schwede and Wenliang Zhang for valuable communications. The authors also thank AIM for hosting the workshop where this question was first discussed, and the main result was proved.

\section{REFERENCES}

[BH] W. Bruns and J. Herzog, Cohen-Macauly rings, Cambridge University Press, 1997 (revised edition).

[F] Hubert Flenner, Divisorenklassengruppen quasihomogener Singularitäten (German), J. Reine Angew. Math. 328 (1981), 128-160, DOI 10.1515/crll.1981.328.128. MR636200 (83a:13009)

[F2] Hubert Flenner, Die Sätze von Bertini für lokale Ringe (German), Math. Ann. 229 (1977), no. 2, 97-111. MR0460317(57 \#311)

[GW] Shiro Goto and Keiichi Watanabe, The structure of one-dimensional F-pure rings, J. Algebra 49 (1977), no. 2, 415-421. MR0453729(56 \#11989)

[He] Stefan Helmke, On Fujita's conjecture, Duke Math. J. 88 (1997), no. 2, 201-216, DOI 10.1215/S0012-7094-97-08807-4. MR1455517 (99e:14003)

[HH] Melvin Hochster and Craig Huneke, Tight closure, invariant theory, and the BriançonSkoda theorem, J. Amer. Math. Soc. 3 (1990), no. 1, 31-116, DOI 10.2307/1990984. MR1017784 (91g:13010)

[Hu] Craig Huneke, Hilbert functions and symbolic powers, Michigan Math. J. 34 (1987), no. 2, 293-318, DOI 10.1307/mmj/1029003560. MR894879 (89b:13037) 
[HS] Craig Huneke and Irena Swanson, Integral closure of ideals, rings, and modules, London Mathematical Society Lecture Note Series, vol. 336, Cambridge University Press, Cambridge, 2006. MR2266432(2008m:13013)

[Li] Joseph Lipman, Rational singularities, with applications to algebraic surfaces and unique factorization, Inst. Hautes Études Sci. Publ. Math. 36 (1969), 195-279. MR.0276239 (43 \#1986)

[LT] Joseph Lipman and Bernard Teissier, Pseudorational local rings and a theorem of BriançonSkoda about integral closures of ideals, Michigan Math. J. 28 (1981), no. 1, 97-116. MR600418 (82f:14004)

[ST] Karl Schwede and Kevin Tucker, On the number of compatibly Frobenius split subvarieties, prime F-ideals, and log canonical centers (English, with English and French summaries), Ann. Inst. Fourier (Grenoble) 60 (2010), no. 5, 1515-1531. MR2766221 (2012d:13007)

[SZ] Karl Schwede and Wenliang Zhang, Bertini theorems for F-singularities, Proc. Lond. Math. Soc. (3) 107 (2013), no. 4, 851-874, DOI 10.1112/plms/pdt007. MR3108833

[St] Richard P. Stanley, The upper bound conjecture and Cohen-Macaulay rings, Studies in Appl. Math. 54 (1975), no. 2, 135-142. MR0458437 (56 \#16640)

[Wa] Keiichi Watanabe, Rational singularities with $k^{*}$-action, Commutative algebra (Trento, 1981), Lecture Notes in Pure and Appl. Math., vol. 84, Dekker, New York, 1983, pp. 339351. MR686954 (84e:14005)

Department of Mathematics, University of Virginia, Charlottesville, Virginia 22904

E-mail address: huneke@virginia.edu

Department of Mathematics, College of Humanities and Sciences, Nihon University, SETAGAYA-KU, TOKYO 156-0045, JAPAN

E-mail address: watanabe@math.chs.nihon-u.ac.jp 